\title{
Hybridization of Expectation-Maximization and K-Means Algorithms for Better Clustering Performance
}

\author{
D. Raja Kishor ${ }^{1}$, N. B. Venkateswarlu ${ }^{2}$ \\ ${ }^{1}$ Dept. of CSE, JNTU, Hyderabad, Telangana, India \\ ${ }^{2}$ Dept. of CSE, AITAM, Tekkali, Andhra Pradesh, India \\ Emails:rajakishor@gmail.com venkat_ritch@yahoo.com
}

Abstract: The present work proposes hybridization of Expectation-Maximization (EM) and K-means techniques as an attempt to speed-up the clustering process. Even though both the $K$-means and EM techniques look into different areas, $K$-means can be viewed as an approximate way to obtain maximum likelihood estimates for the means. Along with the proposed algorithm for hybridization, the present work also experiments with the Standard EM algorithm. Six different datasets, three of which synthetic datasets, are used for the experiments. Clustering fitness and Sum of Squared Errors (SSE) are computed for measuring the clustering performance. In all the experiments it is observed that the proposed algorithm for hybridization of EM and K-means techniques is consistently taking less execution time with acceptable Clustering Fitness value and less SSE than the standard EM algorithm. It is also observed that the proposed algorithm is producing better clustering results than the Cluster package of Purdue University.

Keywords: Hybridization, clustering, K-means, mixture models, expectation maximization, clustering fitness, sum of squared errors.

\section{Introduction}

The Expectation Maximization (EM) algorithm is a model-based clustering technique, which attempts to optimize the fit between the given data and some mathematical model. Such methods are often based on the assumption that the data is generated by a mixture of underlying probability distributions [1].

The EM is an effective, popular technique for estimating mixture model parameters (like cluster weights and means) [7-9]. When compared to other clustering algorithms, the EM algorithm demands more computational efforts although it produces exceptionally good results [20-22]. Many researchers 
experimented on some variants (like Generalized EM (GEM), Expectation Conditional Maximization (ECM), Sparse EM (SpEM), Lazy EM (LEM), Expectation-Conditional Maximization Either (ECME) algorithm and the Space Alternating Generalized Expectation (SAGE) maximization algorithms) in order to reduce the execution time of EM algorithm [17, 18]. In [19], the use of Winograd's algorithm is proposed to reduce the computational efforts of E-step and M-step of the standard EM algorithm. In [15], the use of multi-criteria models is proposed to design clusters with the aim of improved clustering performance. All their experiments aimed at the speed-up of the EM algorithm by yielding the same results as the Standard EM algorithm or better results without sacrificing its simplicity and stability.

As an attempt to speed up the clustering process, the present work proposes the hybridization of EM and K-means algorithms. The K-means algorithm is a very popular algorithm for data clustering, which aims at the local minimum of the distortion $[2,23]$. EM is a model based approach, which aims at finding clusters such that maximum likelihood of each cluster's parameters is obtained. In EM, each observation belongs to each cluster with a certain probability [2]. The K-means algorithm is the 2nd dominantly used data mining algorithm and the EM algorithm is the 5th dominantly used data mining algorithm [3, 4, 24]. Though both K-means and EM techniques look into different areas [2,23], K-means can be viewed as an approximate way to obtain maximum likelihood estimates for the means, which is the goal of density estimation in EM [23, 24].

In the present work, along with the proposed algorithm for hybridization of EM and K-means techniques, experiments are carried out with the standard EM algorithm. In all the experiments, it is observed that the proposed algorithm for hybridization of EM and K-means techniques is consistently taking less execution time to produce the clustering results with acceptable clustering fitness value and less SSE in comparison to the standard EM algorithm. The proposed algorithm is also observed to produce clustering results with better performance than the Cluster Package of Purdue University [26].

\section{The Standard EM (StEM) algorithm}

EM algorithm partitions the given data by calculating the maximum a posteriori principle using the conditional probabilities [17]. Given a guess for the parameter values, the EM algorithm calculates the probability that each point belongs to each distribution and then uses these probabilities to compute a new estimate for the parameter. The EM algorithm iteratively refines initial mixture model parameter estimates to better fit the data and terminates at a locally optimal solution.

The standard EM $[10,11]$ for Gaussian Mixture Models (GMM) assumes that the algorithm will estimate k class distributions $C_{j}, j=1, \ldots, k$. For each of the input vectors $X_{i}, i=1, \ldots, N$, the algorithm calculates the probability $P\left(C_{j} \mid X_{i}\right)$. The highest probability will point to the vector's class. 
The EM algorithm works iteratively by applying two steps: the Expectation step (E-step) and the Maximization step (M-step). Formally, $\theta(t)=\left\{\mu_{j}(t), \sum_{j}(t), W_{j}(t)\right\}, \quad j=1, \ldots, k$, stands for successive parameter estimates.

Given a dataset of $N, d$-dimensional vectors, the EM algorithm has to cluster them into $k$ groups.

The multi-dimensional Gaussian distribution for the cluster $C_{j}$ is parameterized by the $d$-dimensional mean column vector $\mu_{j}$ and $d \mathrm{x} d$ covariance matrix $\Sigma_{j}$ is given as follows [10]:

$$
P\left(X_{i} \mid C_{j}\right)=\frac{1}{\sqrt{(2 \Pi)^{d}\left|\sum_{j}\right|}} e^{-\frac{1}{2}\left(X_{i}-\mu_{j}\right)^{\mathrm{T}}\left(\Sigma_{j}\right)^{-1}\left(X_{i}-\mu_{j}\right)},
$$

where $X_{i}$ is a sample column vector, the superscript $\mathrm{T}$ indicates transpose of a column vector, $\left|\Sigma_{j}\right|$ is the determinant of $\Sigma_{j}$, and $\left(\Sigma_{j}\right)^{-1}$ is its matrix inverse of covariance matrix $\Sigma_{j}$.

The mixture model probability density function [10] is

$$
P\left(X_{i}\right)=\sum_{l=1}^{k} W_{l} P\left(X_{i} \mid C_{j}\right),
$$

where $W_{l}$ is the weight of cluster $C_{l}$.

\subsection{Termination condition}

As the termination condition, percentage change is computed using the following formula:

$$
\text { Percentage change }=\frac{\left|\Psi_{t}-\Psi_{t+1}\right|}{\Psi_{t}} * 100,
$$

where $\Psi_{t}$ is the number of vectors assigned to new clusters in $t$-th iteration, and $\Psi_{t+1}$ is the number of vectors assigned to new clusters in $(t+1)$-th iteration. The symbol $*$ indicates multiplication. The algorithm terminates when the percentage change $<3$. The EM algorithm for Gaussian Mixture Model [10] proceeds as follows:

Step 1. Initialize mixture model parameters: set the current iteration $t=0$; set initial weights, $W$, to $1 / k$ for all $k$ clusters; select $k$ vectors randomly from the dataset as the initial cluster means, $\mu$; compute global covariance matrix for the dataset and set it to be the initial covariance matrix, $\sum$, for all clusters.

Step 2 (E-step). Estimate the probability of each class $C_{j}, j=1,2, \ldots, k$, given a certain vector $X_{i}, i=1,2, \ldots, N$, for current iteration $t$ using the following formula and assign $X_{i}$ to the cluster with the maximum probability,

$$
P\left(C_{j} \mid X_{i}\right)=\frac{W_{j} P\left(X_{i} \mid C_{j}\right)}{P\left(X_{i}\right)}=\frac{\left|\sum_{j}(t)\right|^{-1 / 2} \exp ^{\eta_{j}} \cdot W_{j}(t)}{\sum_{l=1}^{k}\left|\sum_{l}(t)\right|^{-1 / 2} \exp ^{\sigma_{l}} \cdot W_{l}(t)},
$$

where 


$$
\begin{aligned}
\eta_{j} & =-\frac{1}{2}\left(X_{i}-\mu_{j}(t)\right)^{\mathrm{T}} \sum_{j}^{-1}(t)\left(X_{i}-\mu_{j}(t)\right), \\
\sigma_{l} & =-\frac{1}{2}\left(X_{i}-\mu_{l}(t)\right)^{\mathrm{T}} \sum_{l}^{-1}(t)\left(X_{i}-\mu_{l}(t)\right) .
\end{aligned}
$$

Each of the $k$ clusters has its mean $\left(\mu_{j}\right)$ and covariance $\left(\Sigma_{j}\right), j=1,2, \ldots, k ; W_{j}$ is the weight of $j$-th cluster.

Step 3 (M-step). Here, for $j$-th cluster, update the parameter estimation for the iteration $t+1$ as follows:

$$
\begin{gathered}
\mu_{j}(t+1)=\frac{\sum_{i=1}^{N} P\left(C_{j} \mid X_{i}\right) X_{i}}{\sum_{i=1}^{N} P\left(C_{j} \mid X_{i}\right)}, \\
\sum_{j}(t+1)=\frac{\sum_{i=1}^{N} P\left(C_{j} \mid X_{i}\right)\left(X_{i}-\mu_{j}(t)\right)\left(X_{i}-\mu_{j}(t)\right)^{\mathrm{T}}}{\sum_{i=1}^{N} P\left(C_{j} \mid X_{i}\right)}, \\
W_{t}(t+1)=\frac{1}{N} \sum_{i=1}^{N} P\left(C_{j} \mid X_{i}\right) .
\end{gathered}
$$

Step 4. Compute percentage change using (3).

Step 5. Stop the process if the percentage change is $<3$. Otherwise, set $t=t+1$ and repeat the Steps 2 up to 4 with the updated parameters.

\section{Hybridization of EM and HbEMKM algorithms}

Though an effectively used algorithm, the EM suffers from slow convergence as it requires heavily on computational efforts involved in repeated computation of the many parameters like covariance matrices, means and weights of the clusters and repeated computation of the inverses of covariance matrices of the clusters $[3,5,24,25]$. On the other hand, the K-means algorithm can be used to simplify the computation and accelerate convergence as it requires only one parameter to compute, i.e., cluster means [23, 24]. While assigning points to the clusters, the EM maximizes the likelihood and the K-means minimizes the distortion with respect to the clusters [23].

The algorithm for conventional K-means is given below [12].

\section{Algorithm K-means}

Step 1. Select $k$ vectors randomly from the dataset as the initial cluster means, $\mu$. Set the current iteration $t=0$.

Step 2. Repeat.

Step 3. Assign each vector $X_{i}$ from the dataset to its closest cluster mean using Euclidean distance,

$$
\operatorname{dist}\left(X_{i}, \mu_{j}\right)=\sqrt{\sum_{l=1}^{d}\left(x_{i l}-\mu_{l j}\right)^{2}},
$$


where $X_{i}$ is the $i$-th vector in the dataset, $\mu_{j}$ is the mean of the cluster $j$, and $d$ is the number of dimensions of a data point.

Step 4. Re-compute the cluster means and set $t=t+1$.

Step 5. Compute percentage change using (3).

Step 6. Until percentage change is $<3$.

Step 7. End of K-means

The present work, as an attempt to speed up the clustering process, experiments with the Hybridization of EM and K-Means algorithms (HbEMKM). Though both EM and K-means techniques look into different areas [2, 23], $\mathrm{K}$-means can be viewed as an approximate way to obtain maximum likelihood estimates for the means, which is the goal of density estimation in EM [23, 24]. Furthermore, $\mathrm{K}$-means is formally equivalent to EM as K-means is a limiting case of fitting data by a mixture of $k$ Gaussians with identical, isotropic covariance matrices $\left(\Sigma=\sigma^{2} \mathbf{I}\right)$, when the soft assignments of data points to mixture components are hardened to allocate each data point solely to the most likely component [3, 23]. A random space is isotropic if its covariance function depends on distance alone [25]. In practice, there is often some conflation of the two algorithms that K-means is sometimes used in density estimation applications due to its more rapid convergence [23].

Also that selection of initial values is critical for EM, since it most likely converges to local maxima around the initial values as EM uses maximum likelihood [2]. It may be a good practice, if the results of K-means are used as initial parameter values for a subsequent execution of EM for the more exact computations $[23,24]$. The present work also experiments on running the EM algorithm on the results of K-Means algorithm (KMEM).

Along with the proposed algorithm for hybridization of EM and K-means techniques, experiments are carried out with the standard EM algorithm and finally performance comparison is made among the results of all experiments. In all the experiments same termination condition, discussed Section 2.1, is used.

The pseudo code for the algorithm is given below. This algorithm performs clustering using $\mathrm{EM}$ and $\mathrm{K}$-means techniques in the alternative iterations till termination. As part of the maximization step for EM, cluster weights, means and covariance matrices are calculated using the results of K-means step.

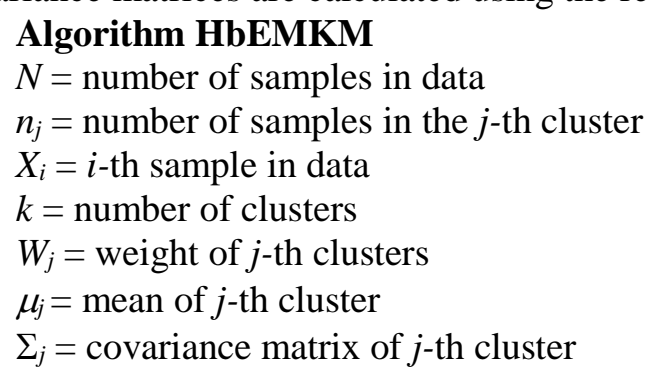

Select $k$ vectors randomly from the input dataset as the initial cluster means, $\mu$.

First, assign each data vector $X_{i}$ to the closest cluster with mean, $\mu_{j}$ using Euclidean distance in the formula (8).

Set isProgress $=$ true 
Repeat while (isProgress $==$ true)

M-Step. Compute means $\mu_{j}$ and covariance matrices $\Sigma_{j}$ for $j=1, \ldots, k$, based on the results of K-Means step.

Compute cluster weights $W_{j}=n_{j} / N$ for $j=1, \ldots, k$.

E-Step. For each given data vector $X_{i}(i=1,2, \ldots, N)$, compute the cluster probability $P\left(C_{j} \mid X_{i}\right)$ for $j=1, \ldots, k$, using (4).

Assign $X_{i}$ to the cluster with $\operatorname{Max}_{j}\left\{P\left(C_{j} \mid X_{i}\right) ; j=1, \ldots, k\right\}$.

Compute percentage change using (3).

IF (percentage change $>=3$ )

Compute cluster means $\mu_{j}$ for $j=1, \ldots, k$, using (5).

K-Means Step. Assign each data vector $X_{i}$ to the closest cluster with mean, $\mu_{j}$ using Euclidean distance in the formula (8).

Compute percentage change using (3).

IF (percentage change $>=3$ )

ELSE

Set isProgress $=$ true

End of inner IF

Set $i$ Progress $=$ false

ELSE

Set isProgress $=$ false

End of outer IF

End of Repeat Loop

End of HbEMKM

\section{Clustering performance measure}

As a measure of clustering performance, the Clustering Fitness [13] is computed. The calculation of Clustering Fitness involves intra-cluster similarity, inter-cluster similarity, and the experiential knowledge, $\lambda$. The main objective of any clustering algorithm is to generate clusters with higher intra-cluster similarity and lower intercluster similarity [16]. So both the measures are taken into consideration for computing Clustering Fitness. The computation of Clustering Fitness results in higher values when the inter-cluster similarity is low and results in lower values for when the inter-cluster similarity is high. To make the computation of Clustering Fitness unbiased, the value of $\lambda$ is taken as 0.5 [13].

\subsection{Intracluster similarity for the cluster $C_{j}$}

It can be quantified via some function of the reciprocals of intracluster radii within each of the resulting clusters. The intracluster similarity of a cluster $C_{j}, 1=j=k$, denoted as $S_{\text {tra }}\left(C_{j}\right)$, is defined by

$$
S_{\text {tra }}\left(C_{j}\right)=\frac{1+n_{j}}{1+\sum_{1}^{n_{j}} \operatorname{dist}\left(I_{l} \text { Centroid }\right)} .
$$


Here, $n_{j}$ is the number of items in cluster $C_{j}, 1=l=n_{j}, I_{l}$ is the $l$-th item in cluster $C_{l}$, and $\operatorname{dist}\left(I_{l}\right.$, Centroid) calculates the distance between $I_{l}$ and the centroid of $C_{j}$, which is the intracluster radius of $C_{j}$. To smooth the value of $S_{\text {tra }}\left(C_{j}\right)$ and allow for possible singleton clusters 1 is added to the denominator and numerator.

\subsection{Intracluster similarity for one clustering result $C$}

Denoted as $S_{\text {tra }}(C)$, Intracluster similarity for one clustering result $C$ is defined by

$$
S_{\text {tra }}(c)=\frac{\sum_{1}^{k} S_{\text {tra }}\left(C_{j}\right)}{k} .
$$

Here, $k$ is the number of resulting clusters in $C$.

\subsection{Intercluster similarity}

It can be quantified via some function of the reciprocals of intercluster radii of the clustering centroids. The intercluster similarity for one of the possible clustering results $C$, denoted as $S_{\text {ter }}(C)$, is defined by

$$
S_{\text {ter }}(C)=\frac{1+n}{1+\sum_{1}^{k} \operatorname{dist}\left(\text { Centroid }_{j}, \text { Centroid }^{2}\right)} .
$$

Here, $k$ is the number of resulting clusters in $C, 1=j=k$, Centroid ${ }_{j}$ is the centroid of the $j$-th cluster in $C$, Centroid ${ }^{2}$ is the centroid of all centroids of clusters in $C$. We compute intercluster radius of Centroid $_{j}$ by calculating $\operatorname{dist}\left(\right.$ Centroid $_{j}$, Centroid $\left.{ }^{2}\right)$, which is distance between Centroid ${ }_{j}$, and Centroid ${ }^{2}$. To smooth the value of $S_{\text {ter }}(C)$ and allow for possible all-inclusive clustering result, 1 is added to the denominator and the numerator.

\subsection{Clustering fitness}

The Clustering Fitness (CF) for one of the possible clustering results $C$ is defined by

$$
\mathrm{CF}=\lambda * S_{\text {tra }}+\frac{1-\lambda}{S_{\text {ter }}} .
$$

Here, $0<\lambda<1$ is an experiential weight. The symbol $*$ indicates multiplication. The present work considers $\lambda=0.5$.

\subsection{Sum of squared errors}

In the present work, Sum of Squared Errors (SSE) is also computed for all the clustering results to measure the clustering performance [6]. The clustering performance is considered to be good if the corresponding SSE is less when compared to the other clustering techniques. The SSE is computed using the following formula

$$
\mathrm{SSE}=\frac{1}{N} \sum_{j=1}^{k} \sum_{X_{i} \in C_{j}}\left|X_{i}-\mu_{j}\right| .
$$


Here, $X_{i}$ is a vector from the dataset, $\mu_{j}$ is the means of the cluster $C_{j}, k$ is the number of clusters and $N$ is the number of vectors in the dataset. $\left|X_{i}-\mu_{j}\right|$ denotes the distance between $X_{i}$ and $\mu_{j}$. The objective of clustering is to minimize the withincluster sum of squared errors. The lesser the SSE, the better the goodness of fit is.

\section{Experiment and results}

Experiments are carried out on the system with Intel(R) Core i7-3770 K with $3.50 \mathrm{GHz}$ processor speed, 8GB RAM with 1666FSB, Windows 7 OS and using JDK1.7.0_45. Separate modules are written for each algorithm to observe the CPU time for clustering any dataset by keeping the same cluster seeds for all methods. $\mathrm{I} / \mathrm{O}$ operations are eliminated and time observed is strictly for clustering of the data (Table 1).

Magic Gamma, Poker Hand, and Letter Recognition datasets are used for the present work from UCI ML dataset repository [14]. An important issue in evaluating data analysis algorithms is the availability of representative data. When real-life data is hard to obtain or when its properties are hard to modify for testing various algorithms, synthetic data becomes an appealing alternative. The present work also uses three synthetic datasets that are generated by an algorithm for generating multivariate normal random variables [27]. The first synthetic dataset is generated assuming all clusters have different means and different covariance matrices. The second synthetic dataset is generated assuming some clusters have the same mean but different covariance matrices. The third synthetic dataset is generated assuming some clusters have the same covariance matrix but different means.

Table 1. Datasets

\begin{tabular}{|l|c|c|}
\hline \multicolumn{1}{|c|}{ Data set } & Number of points & Number of dimensions \\
\hline Letter Recognition Data & 20,000 & 16 \\
\hline Magic Gamma data & 19,020 & 10 \\
\hline Poker Hand data & $1,025,010$ & 10 \\
\hline Synthetic data-1 & 50,000 & 10 \\
\hline Synthetic data-2 & 50,000 & 10 \\
\hline Synthetic data-3 & 50,000 & 10 \\
\hline
\end{tabular}

All the algorithms are studied by executing on each dataset by varying number of clusters (i.e., $k=10,11,12,13,14,15$ ). The details of execution time, clustering fitness and SSE of each algorithm are separately given in the tables below for each dataset.

\subsection{Observations on letter recognition dataset}

The Tables 2, 3 and 4 consist of the execution time, the cluster fitness and Sum of Squared Error (SSE), respectively, of algorithms discussed in sections 2 and 3 and the Cluster Package of Purdue University performed on Letter Recognition dataset. The observations are also shown in the Figs 1, 2 and 3. 
Table 2. Execution time of each clustering method (s)

\begin{tabular}{|c|c|c|c|c|}
\hline$K$ & StEM & KMEM & HbEMKM & Cluster 3.6.7 \\
\hline 10 & 16.4120 & 5.9210 & 0.2440 & 17.5760 \\
\hline 11 & 6.4330 & 11.4470 & 0.3360 & 20.0250 \\
\hline 12 & 14.0750 & 6.5540 & 0.4930 & 26.4340 \\
\hline 13 & 7.6010 & 5.8790 & 0.3880 & 24.1890 \\
\hline 14 & 5.1380 & 8.9930 & 0.4240 & 30.7420 \\
\hline 15 & 13.5860 & 6.1920 & 0.7500 & 41.3680 \\
\hline
\end{tabular}

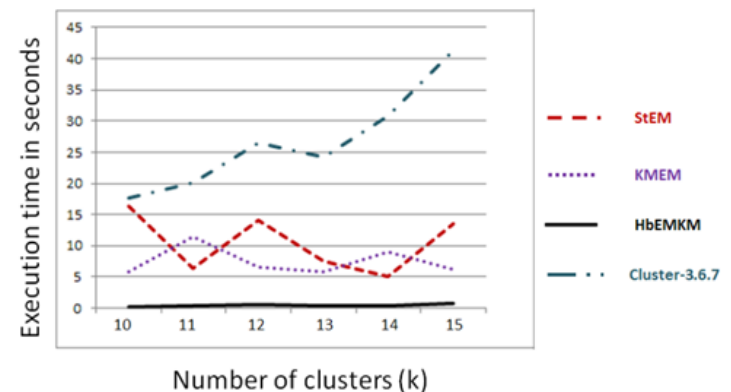

Fig. 1. Letter recognition dataset: Execution times

Table 3. Clustering fitness of each clustering method

\begin{tabular}{|c|c|c|c|c|}
\hline$K$ & StEM & KMEM & HbEMKM & Cluster 3.6.7 \\
\hline 10 & 2.6258 & 2.7286 & 2.9425 & 2.7812 \\
\hline 11 & 2.6063 & 2.9516 & 3.1282 & 2.7391 \\
\hline 12 & 2.7610 & 2.8908 & 3.1003 & 2.7088 \\
\hline 13 & 2.8867 & 3.1064 & 3.2270 & 2.9507 \\
\hline 14 & 3.0719 & 3.3224 & 3.4473 & 2.8795 \\
\hline 15 & 2.9324 & 3.0599 & 3.3460 & 3.0419 \\
\hline
\end{tabular}

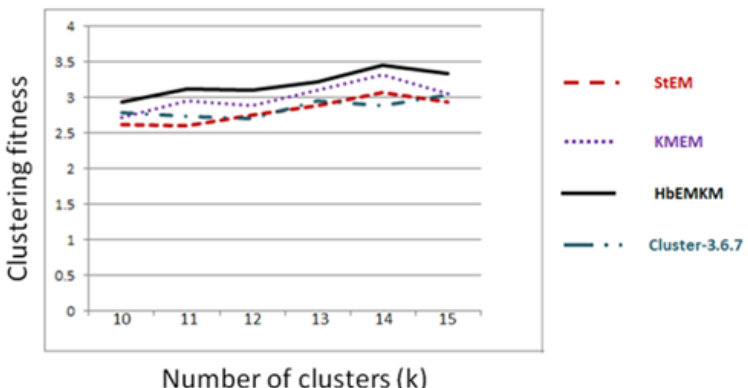

Fig. 2. Letter recognition dataset: Clustering fitness

Table 4. SSE of each clustering method

\begin{tabular}{|c|c|c|c|c|}
\hline$K$ & StEM & KMEM & HbEMKM & Cluster 3.6.7 \\
\hline 10 & 58.31780253 & 54.90029903 & 46.79034965 & 59.54343871 \\
\hline 11 & 56.81365607 & 50.53051120 & 43.76738443 & 59.06663898 \\
\hline 12 & 55.26046620 & 49.59847946 & 43.60821184 & 55.55461758 \\
\hline 13 & 52.24618292 & 47.06660999 & 41.57619654 & 54.98634779 \\
\hline 14 & 52.43310568 & 46.24255323 & 39.85697243 & 53.61458606 \\
\hline 15 & 52.74977604 & 47.52093477 & 39.76810321 & 51.85401078 \\
\hline
\end{tabular}




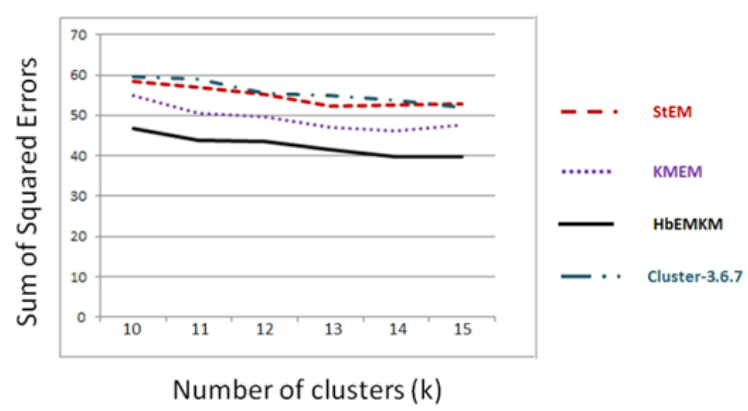

Fig. 3. Letter recognition dataset: Sum of squared errors

\subsection{Observations on magic gamma dataset}

Tables 5, 6 and 7 consist of the execution time, the cluster fitness and Sum of Squared Error (SSE), respectively, of algorithms discussed in Sections 2 and 3 and the Cluster Package of Purdue University performed on Magic Gamma dataset. The observations are also shown in the Figs 4, 5 and 6.

Table 5. Execution time of each clustering method (s)

\begin{tabular}{|c|c|c|c|c|}
\hline$K$ & StEM & KMEM & HbEMKM & Cluster 3.6.7 \\
\hline 10 & 3.5360 & 0.7920 & 0.1830 & 7.2890 \\
\hline 11 & 3.7360 & 4.0920 & 0.1110 & 10.2680 \\
\hline 12 & 3.2410 & 3.1720 & 0.2420 & 9.3120 \\
\hline 13 & 3.2390 & 4.9610 & 0.2570 & 11.0620 \\
\hline 14 & 6.8540 & 7.9350 & 0.4070 & 9.5590 \\
\hline 15 & 5.9050 & 3.3490 & 0.5020 & 18.6780 \\
\hline
\end{tabular}

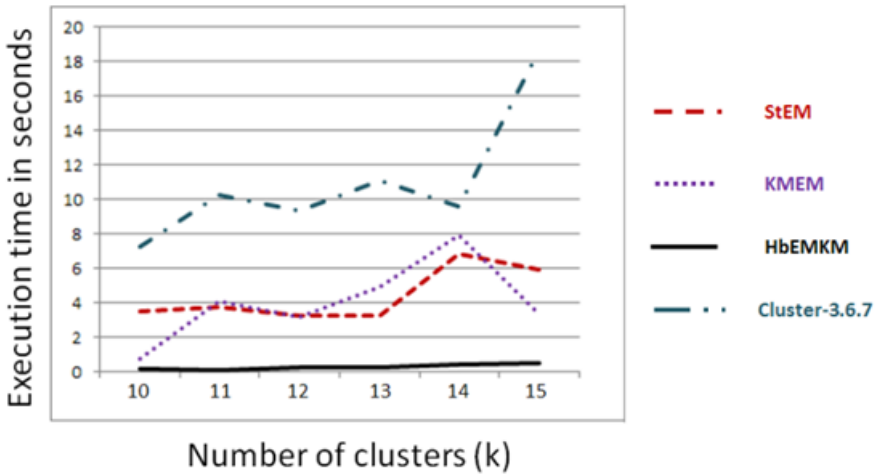

Fig. 4. Magic gamma dataset: Execution times

Table 6. Clustering fitness of each clustering method

\begin{tabular}{|c|c|c|c|c|}
\hline$K$ & StEM & KMEM & HbEMKM & Cluster 3.6.7 \\
\hline 10 & 29.8434 & 46.1219 & 50.8960 & 34.9529 \\
\hline 11 & 35.9602 & 40.2215 & 46.6300 & 36.0443 \\
\hline 12 & 37.5347 & 44.2417 & 57.5562 & 38.4638 \\
\hline 13 & 34.8555 & 41.3342 & 52.9504 & 40.7634 \\
\hline 14 & 33.7390 & 39.9490 & 52.9743 & 41.0526 \\
\hline 15 & 42.8234 & 46.1663 & 61.7020 & 39.9385 \\
\hline
\end{tabular}




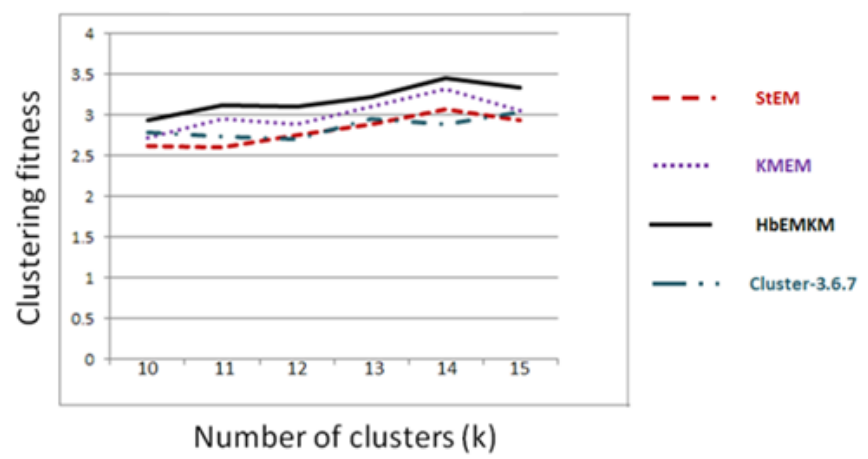

Fig. 5. Magic gamma dataset: Clustering fitness

Table 7. SSE of each clustering method

\begin{tabular}{|c|c|c|c|c|}
\hline$K$ & StEM & KMEM & HbEMKM & Cluster 3.6.7 \\
\hline 10 & 10235.04311 & 6336.868507 & 5116.794358 & 9619.032540 \\
\hline 11 & 9578.544208 & 8140.627114 & 5013.559560 & 8606.290689 \\
\hline 12 & 9873.706092 & 7544.572959 & 4409.876943 & 8838.627792 \\
\hline 13 & 9272.422300 & 8045.302214 & 4658.203266 & 8743.184190 \\
\hline 14 & 9398.092913 & 7830.483924 & 4397.280683 & 8191.174528 \\
\hline 15 & 8646.400371 & 6528.632337 & 3803.336041 & 8620.268594 \\
\hline
\end{tabular}

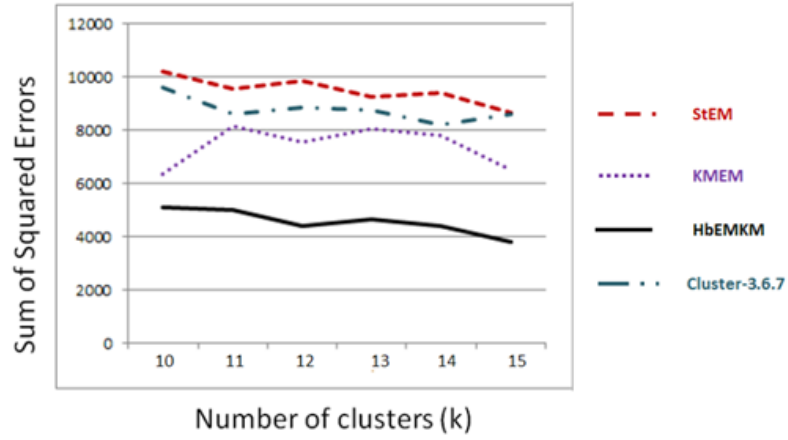

Fig. 6. Magic gamma dataset: Sum of squared errors

\subsection{Observations on Poker hand dataset}

The tables 8, 9 and 10 consist of the execution time, the cluster fitness and Sum of Squared Error (SSE), respectively, of algorithms discussed in Sections 2 and 3 and the Cluster Package of Purdue University performed on Poker Hand dataset. The observations are also shown in Figs 7, 8 and 9.

Table 8. Execution time of each clustering method (s)

\begin{tabular}{|c|c|c|c|c|}
\hline$K$ & StEM & KMEM & HbEMKM & Cluster 3.6.7 \\
\hline 10 & 66.1490 & 224.7950 & 15.5420 & 1016.9140 \\
\hline 11 & 74.0110 & 107.9470 & 23.3930 & 2557.5940 \\
\hline 12 & 78.4610 & 82.4270 & 31.0430 & 3328.3360 \\
\hline 13 & 85.2930 & 72.0570 & 29.0900 & 3434.1700 \\
\hline 14 & 91.0140 & 332.3750 & 46.2370 & 3160.3870 \\
\hline 15 & 117.2070 & 238.8700 & 28.6360 & 2809.5350 \\
\hline
\end{tabular}




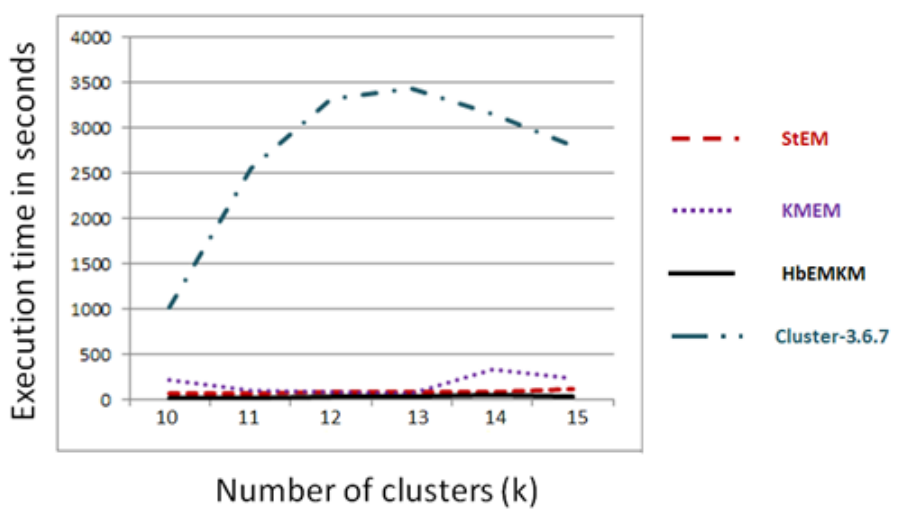

Fig. 7. Poker hand dataset: Execution times

Table 9. Clustering fitness of each clustering method

\begin{tabular}{|c|c|c|c|c|}
\hline$K$ & StEM & KMEM & HbEMKM & Cluster 3.6.7 \\
\hline 10 & 1.3840 & 2.7620 & 2.8951 & 1.2293 \\
\hline 11 & 1.6828 & 2.8351 & 2.9882 & 1.8606 \\
\hline 12 & 1.5570 & 2.9154 & 3.0631 & 1.3815 \\
\hline 13 & 1.4044 & 2.9795 & 3.1120 & 1.0973 \\
\hline 14 & 1.5571 & 3.0186 & 3.1739 & 1.5624 \\
\hline 15 & 1.8413 & 3.0663 & 3.2148 & 1.0539 \\
\hline
\end{tabular}

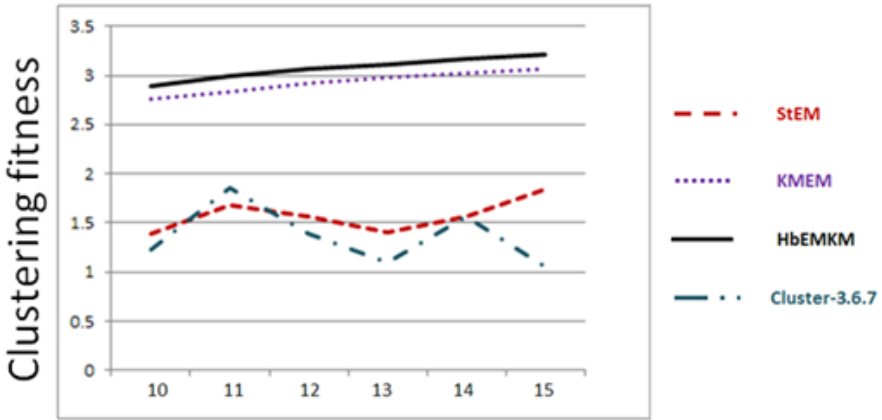

Number of clusters ( $k$ )

Fig. 8. Poker hand dataset: Clustering fitness

Table 10. SSE of each clustering method

\begin{tabular}{|c|c|c|c|c|}
\hline$K$ & StEM & KMEM & HbEMKM & Cluster 3.6.7 \\
\hline 10 & 61.89049571 & 40.41746868 & 39.33511213 & 72.07099026 \\
\hline 11 & 57.49226138 & 38.17264347 & 37.30469352 & 62.23416768 \\
\hline 12 & 59.57113812 & 36.38456914 & 35.82492696 & 70.92287882 \\
\hline 13 & 61.55035153 & 35.23419731 & 34.83422455 & 72.85375279 \\
\hline 14 & 58.56628704 & 34.80452906 & 33.53945731 & 73.87162564 \\
\hline 15 & 56.33032095 & 33.82117461 & 32.75190094 & 71.93356843 \\
\hline
\end{tabular}




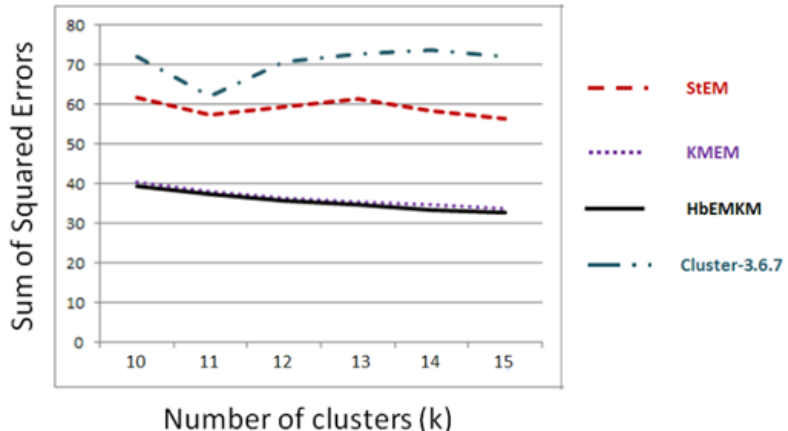

Fig. 9. Poker hand dataset: Sum of squared errors

\subsection{Observations on Synthetic dataset-1}

Tables 11, 12 and 13 consist of the execution time, the cluster fitness and SSE, respectively, of algorithms discussed in Sections 2 and 3 and the Cluster Package of Purdue University performed on Synthetic dataset-1. The observations are also shown in Figs 10, 11 and 12.

Table 11. Execution time of each clustering method (s)

\begin{tabular}{|c|c|c|c|c|}
\hline$K$ & StEM & KMEM & HbEMKM & Cluster 3.6.7 \\
\hline 10 & 3.8230 & 3.3670 & 0.6580 & 43.3850 \\
\hline 11 & 5.6100 & 2.9100 & 0.8740 & 46.5140 \\
\hline 12 & 6.1160 & 3.9880 & 0.9400 & 59.1600 \\
\hline 13 & 4.9520 & 4.2760 & 1.3410 & 70.7840 \\
\hline 14 & 4.4550 & 3.7160 & 1.0860 & 87.9630 \\
\hline 15 & 4.7690 & 6.8270 & 0.9680 & 106.1600 \\
\hline
\end{tabular}

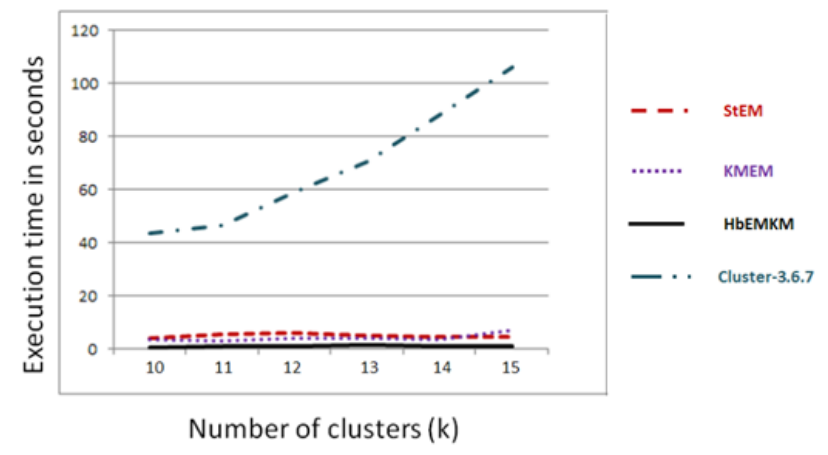

Fig. 10. Synthetic dataset-1: Execution times

Table 12. Clustering fitness of each clustering method

\begin{tabular}{|c|c|c|c|c|}
\hline$K$ & StEM & KMEM & HbEMKM & Cluster 3.6.7 \\
\hline 10 & 582.8037 & 989.8852 & 1070.4906 & 862.1022 \\
\hline 11 & 719.8391 & 1002.2908 & 1080.2918 & 901.5273 \\
\hline 12 & 690.8997 & 1043.5350 & 1135.5673 & 897.9433 \\
\hline 13 & 663.8376 & 1049.8692 & 1143.5548 & 380.6306 \\
\hline 14 & 763.8713 & 1081.2933 & 1165.1284 & 443.3503 \\
\hline 15 & 842.4782 & 1107.4315 & 1200.3491 & 361.4750 \\
\hline
\end{tabular}




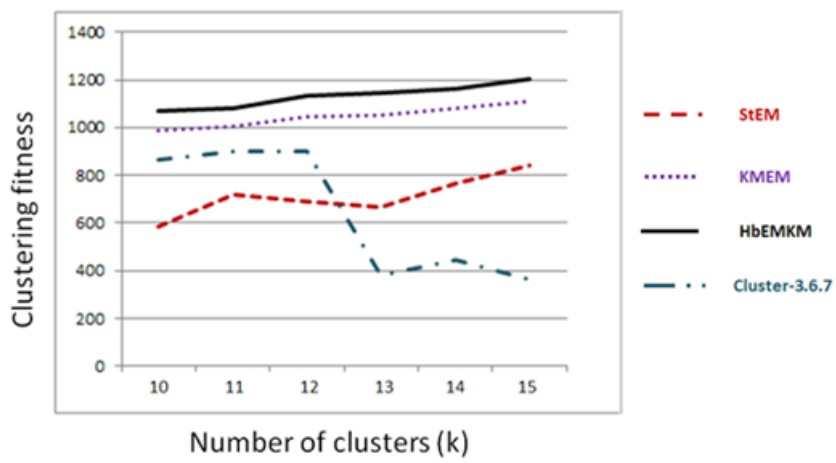

Fig. 11. Synthetic dataset-1: Clustering fitness

Table 13. SSE of each clustering method

\begin{tabular}{|c|c|c|c|c|}
\hline$K$ & StEM & KMEM & HbEMKM & Cluster 3.6.7 \\
\hline 10 & 14628710.78 & 11859786.85 & 11684878.89 & 14388425.51 \\
\hline 11 & 14386916.86 & 11658179.86 & 11468010.80 & 13455719.67 \\
\hline 12 & 13874673.81 & 11491360.59 & 11251086.05 & 13535376.15 \\
\hline 13 & 14099367.22 & 11385248.64 & 11110137.28 & 20481277.55 \\
\hline 14 & 13589123.08 & 11212795.22 & 11046519.80 & 25434272.93 \\
\hline 15 & 13452374.50 & 11267402.97 & 10862782.33 & 21592712.96 \\
\hline
\end{tabular}

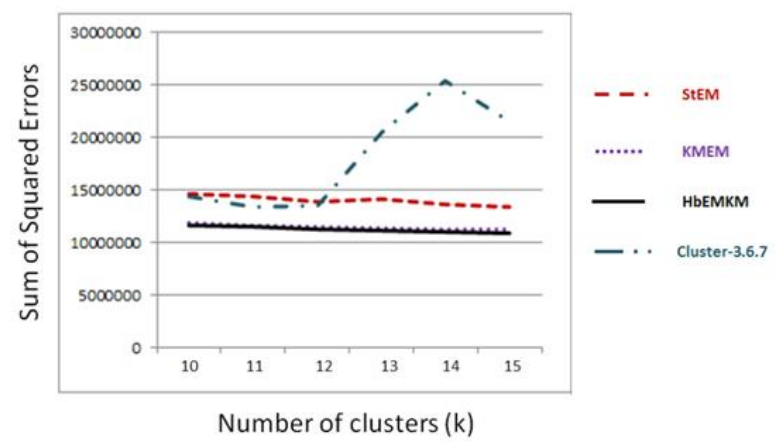

Fig. 12. Synthetic dataset-1: Sum of squared errors

5.5. Observations on synthetic dataset-2

Tables 14, 15 and 16 consist of the execution time, the cluster fitness and Sum of Squared Error (SSE), respectively, of algorithms discussed in sections 2 and 3 and the Cluster Package of Purdue University performed on Synthetic dataset-2. The observations are also shown in Figs 13, 14 and 15.

Table 14. Execution time of each clustering method (s)

\begin{tabular}{|c|c|c|c|c|}
\hline$K$ & StEM & KMEM & HbEMKM & Cluster 3.6.7 \\
\hline 10 & 3.8420 & 2.6790 & 0.6660 & 30.8250 \\
\hline 11 & 4.9020 & 2.9210 & 0.8630 & 51.8800 \\
\hline 12 & 4.5760 & 3.9120 & 0.9290 & 49.4800 \\
\hline 13 & 7.4250 & 5.1100 & 1.0130 & 64.8300 \\
\hline 14 & 7.1170 & 3.6620 & 1.0750 & 87.2220 \\
\hline 15 & 5.7130 & 3.9850 & 1.1530 & 88.0840 \\
\hline
\end{tabular}




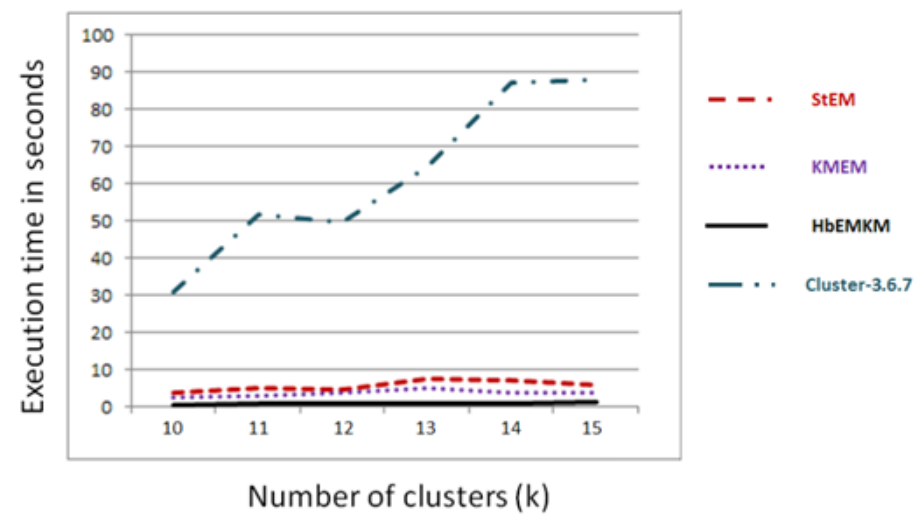

Fig. 13. Synthetic dataset-2: Execution times

Table 15. Clustering fitness of each clustering method

\begin{tabular}{|c|c|c|c|c|}
\hline$K$ & StEM & KMEM & HbEMKM & Cluster 3.6.7 \\
\hline 10 & 592.9165 & 985.5797 & 1064.7669 & 479.4660 \\
\hline 11 & 705.1030 & 998.8906 & 1083.0545 & 505.4192 \\
\hline 12 & 616.7459 & 1028.0205 & 1071.5066 & 391.3777 \\
\hline 13 & 865.7343 & 1040.6712 & 1148.6615 & 435.4822 \\
\hline 14 & 761.9546 & 1080.9615 & 1174.8407 & 321.3893 \\
\hline 15 & 775.9452 & 1096.7161 & 1180.8662 & 412.5189 \\
\hline
\end{tabular}

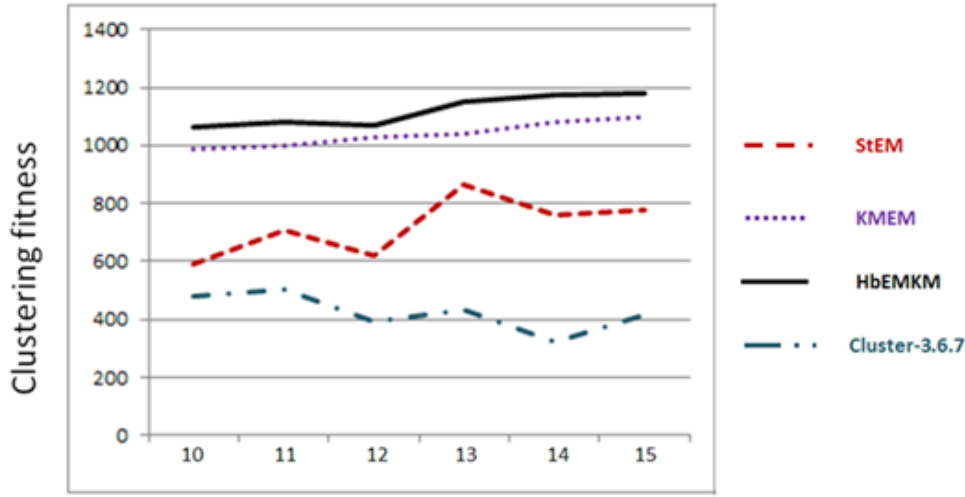

Number of clusters (k)

Fig. 14. Synthetic dataset-2: Clustering fitness

Table 16. SSE of each clustering method

\begin{tabular}{|c|c|c|c|c|}
\hline$K$ & StEM & KMEM & HbEMKM & Cluster 3.6.7 \\
\hline 10 & 14439664.00 & 11760241.27 & 11598373.61 & 20164991.50 \\
\hline 11 & 13902439.10 & 11528187.47 & 11355916.26 & 21276089.23 \\
\hline 12 & 14232153.20 & 11421357.02 & 11287930.24 & 21633831.68 \\
\hline 13 & 13415780.69 & 11319653.67 & 11024847.95 & 21181605.38 \\
\hline 14 & 13336198.27 & 11100700.03 & 10882717.53 & 23815685.30 \\
\hline 15 & 13320932.07 & 10941351.39 & 10743553.26 & 21087020.41 \\
\hline
\end{tabular}




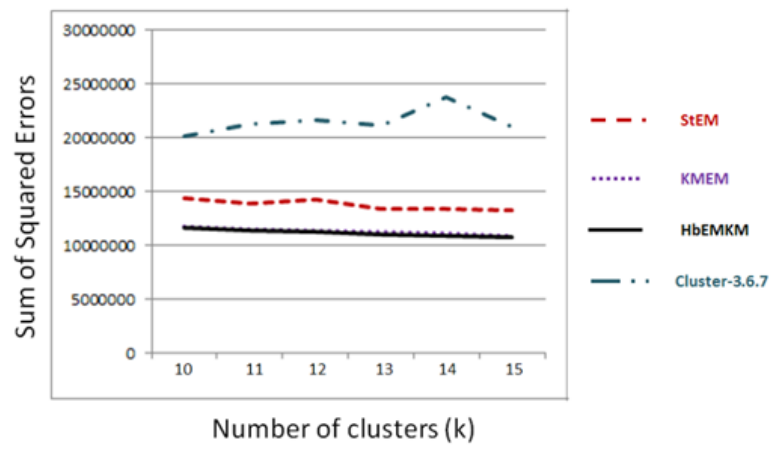

Fig. 15. Synthetic dataset-2: Sum of squared errors

5.6. Observations on Synthetic dataset-3

Tables 17, 18 and 19 consist of the execution time, the cluster fitness and SSE, respectively, of algorithms discussed in Sections 2 and 3 and the Cluster Package of Purdue University performed on Synthetic dataset-3. The observations are also shown in Figs 16, 17 and 18.

Table 17. Execution time of each clustering method (s)

\begin{tabular}{|c|c|c|c|c|}
\hline$K$ & StEM & KMEM & HbEMKM & Cluster 3.6.7 \\
\hline 10 & 8.2650 & 2.0130 & 0.8030 & 31.9960 \\
\hline 11 & 4.1960 & 3.6610 & 0.7170 & 60.8990 \\
\hline 12 & 4.5890 & 2.4560 & 0.7870 & 48.1750 \\
\hline 13 & 5.7860 & 4.2670 & 1.0130 & 70.2460 \\
\hline 14 & 6.2350 & 8.0820 & 0.7250 & 62.2360 \\
\hline 15 & 5.7190 & 4.9670 & 0.9640 & 95.2090 \\
\hline
\end{tabular}

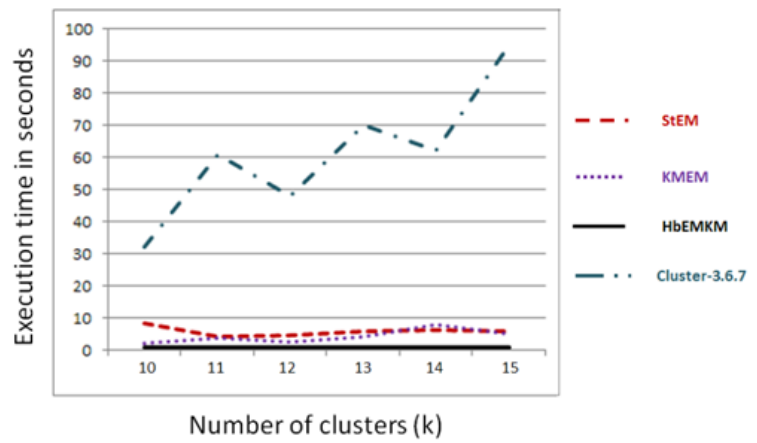

Fig. 16. Synthetic dataset-3: Execution times

Table 18. Clustering fitness of each clustering method

\begin{tabular}{|c|c|c|c|c|}
\hline$K$ & StEM & KMEM & HbEMKM & Cluster 3.6.7 \\
\hline 10 & 623.6250 & 972.4719 & 1048.2655 & 500.3920 \\
\hline 11 & 671.4153 & 982.1240 & 1066.9712 & 391.8017 \\
\hline 12 & 670.1226 & 1026.8664 & 1106.1198 & 419.4653 \\
\hline 13 & 797.2359 & 1039.7200 & 1116.5491 & 372.0694 \\
\hline 14 & 872.9962 & 1048.6642 & 1157.4691 & 397.6201 \\
\hline 15 & 685.2117 & 1075.6261 & 1149.7448 & 332.1400 \\
\hline
\end{tabular}




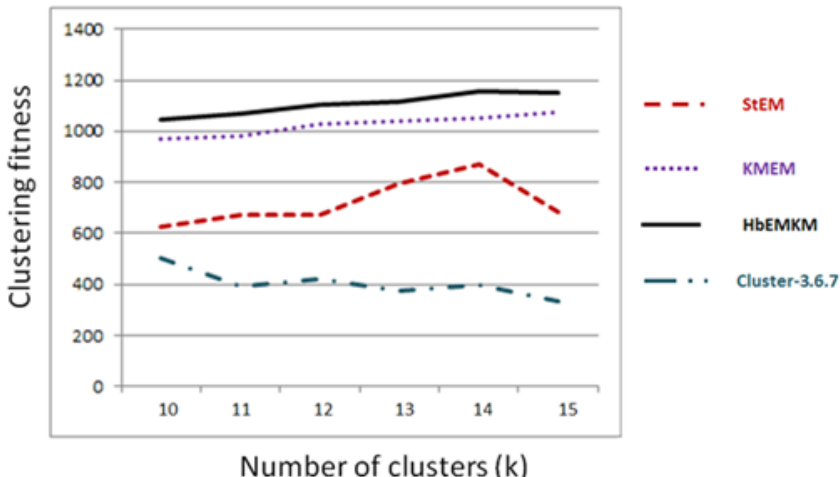

Fig. 17. Synthetic dataset-3: Clustering fitness

Table 19. SSE of each clustering method

\begin{tabular}{|c|c|c|c|c|}
\hline$K$ & StEM & KMEM & HbEMKM & Cluster 3.6.7 \\
\hline 10 & 13456811.43 & 11348281.15 & 11197451.26 & 19005111.06 \\
\hline 11 & 13810971.74 & 11258784.69 & 11072963.24 & 19915617.9 \\
\hline 12 & 13190851.64 & 10946003.08 & 10842930.4 & 19915392.35 \\
\hline 13 & 13235505.84 & 10976305.12 & 10717404.62 & 21071287.76 \\
\hline 14 & 12527770.20 & 11236068.19 & 10616547.71 & 19920287.74 \\
\hline 15 & 13107521.88 & 10636008.11 & 10406779.38 & 21017062.74 \\
\hline
\end{tabular}

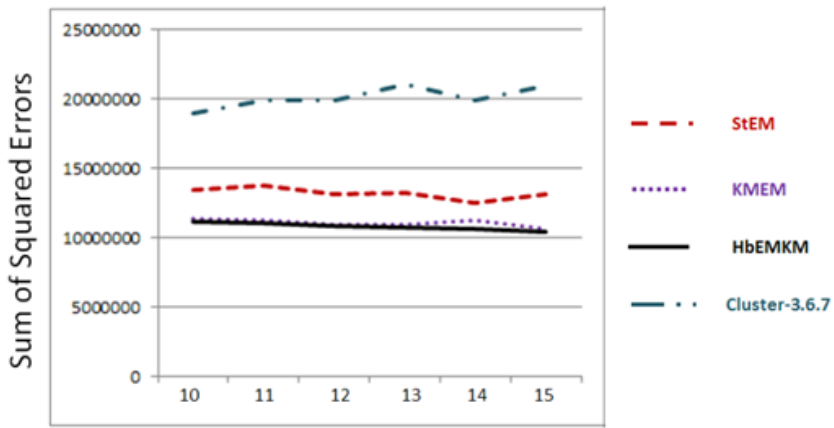

Number of clusters ( $\mathrm{k}$ )

Fig. 18. Synthetic dataset-3: Sum of squared errors

\section{Conclusion}

The proposed algorithm for Hybridization of EM and K-means is consistently taking less computational time with all the tested datasets. The algorithm also takes less computational time when compared to the Cluster-3.6.7 package from Purdue University. The proposed algorithm also produces the results with higher clustering fitness values than the other algorithms including Cluster-3.6.7. It is also observed that the proposed algorithm produces the clustering results with lesser SSE values than the other algorithms including the Cluster-3.6.7 package. Therefore, the present work proposes Hybridization of EM and K-means algorithms as a faster clustering technique with improved performance. 


\section{References}

1. Fraley, C., A. E. R a ftery. Model-Based Clustering, Discriminant Analysis, and Density Estimation. - Journal of the American Statistical Association, Vol. 97, 2002, No 458, p. 611.

2. Ade bi si, A. A., O. E. Olus a yo, O. S. Olatund e. An Exploratory Study of K-Means and Expectation Maximization Algorithms. - British Journal of Mathematics \& Computer Science, Vol. 2, 2012, No 2, pp. 62-71.

3. W u, X., V. K u m a r, J. R. Qu in l a n, J. Ghos h, Q. Y a n g, H. Motod a, G. J. M c L a c h 1 a n, A. N g, B. Li u, P. S. Y u, Z.-H. Z hou, M. S t e in b a c h, D. J. H and, D. S t e in ber g. Survey Paper: Top 10 Algorithms in Data Mining. - Knowledge and Information Systems, Vol. 14, 2008, pp. 1-37.

4. M a c Q u e e n, J. Some Methods for Classification and Analysis of Multivariate Observations. - In: Proc. of 5th Berkeley Symposium on Mathematics, Statistics and Probability, Vol. 1, 1967, pp. 281-296.

5. McLachla n, G. J., T. Krishnan. The EM Algorithm and Extensions, 2/e. John Wiley \& Sons, Inc., 2007.

6. $\mathrm{H}$ a n, J., M. K a m b e r. Data Mining Concepts and Techniques, 2/e. New Delhi, India, Elsevier, Inc., 2007.

7. T a n, P.-N., M. S t e i n b a c h, V. K u m a r. Introduction to Data Mining, 1/e. Pearson Education, 2007.

8. Yeung, K. Y., C. Fraley, A. Murua, A. E. Raftery, W. L. Ruzzo. Model-Based Clustering and Data Transformations for Gene Expression Data. - Bioinformatics, Vol. 17, 2010, No 10, pp. 977-987.

9. B radley, P. S., U. M. Fay y ad, C. A. Re in a. Scaling EM (Expectation-Maximization) Clustering to Large Databases. Technical Report, Microsoft Research, MSR-TR-98-35, 1999.

10. Körting, T. S., L. V. Dutra, L. M. G. Fon s e c a, G. J. Erth a l. Assessment of a Modified Version of the EM Algorithm for Remote Sensing Data Classification. - In: Proc. of Iberoamerican Congress on Pattern Recognition (CIARP). São Paulo, Brazil, LNCS 6419, 2010, pp. 476-483.

11. Körting, T. S., L. V. Dutra, L. M. G. Fonseca, G. Erthal, F. C. da Silva. Improvements to Expectation-Maximization Approach for Unsupervised Classification of Remote Sensing Data. GeoINFO, Campos do Jordão, SP, Brazil, 2007.

12. Agg a rw a 1, N., K. A g g a rw a 1. A Mid-Point Based K-Mean Clustering Algorithm for Data Mining. - International Journal on Computer Science and Engineering, Vol. 4, 2012, No 6, pp. 1174-1180.

13. H a n, X., T. Z h a o. Auto-K Dynamic Clustering Algorithm. - Journal of Animal and Veterinary Advances, Vol. 4, 2005, No 5, pp. 535-539.

14. UCL Machine Learning Repository http://archive.ics.uci.edu/ml/datasets.html

15. Radeva, I. Multi-Criteria Models for Clusters Design. - Cybernetics and Information Technology, Vol. 13, 2013, No 1, pp. 18-33.

16. R a o, V. S. H., M. V. Jo n n a la gedd a. Insurance Dynamics - A Data Mining Approach for Customer Retention in Health Care Insurance Industry. - Cybernetics and Information Technologies, Vol. 12, 2012, No 1, pp. 49-60.

17. Jollois, F. X., M. Nadif. Speed-up for the Expectation-Maximization Algorithm for Clustering Categorical Data. - J. Glob Optim, Vol. 37, 2007, pp. 513-525.

18. M e n g, X.-L., D. V a n D y k. The EM Algorithm - An Old Folk-Song Sung to a Fast New Tune. - Journal of the Royal Statistical Society, Vol. 59, 1997, No 3, pp. 511-567.

19. N a ge ndra, K. D. J., J. V. R. Murthy, N. B. Ve nkat e s warlu. Fast Expectation Maximization Clustering Algorithm. - International Journal of Computational Intelligence Research, Vol. 8, 2012, No 2, pp. 71-94.

20. Jollois, F.-X., M. Nadif. Speed-up for the Expectation-Maximization Algorithm for Clustering Categorical Data. - Journal of Global Optimization, Vol. 37, 2007, No 4, pp. 513-525. 
21. N e a 1, R., G. E. H i n t o n. A View of the EM Algorithm That Justifies Incremental, Sparse, and Other Variants, Learning in Graphical Models. MA, USA, Kluwer Academic Publishers, 1998.

22. X u, R., D. W u n s h II. Survey of Clustering Algorithms. - IEEE Transactions on Neural Networks, Vol. 16, 2005, No 3, pp. 645-678.

23. Ke arn s, M., Y. Man s our, A. Ng. An Information-Theoretic Analysis of Hard and Soft Assignment Methods for Clustering, Uncertainty in Artificial Intelligence. - In: Proc. of 13th Conference Annual Conference on Uncertainty in Artificial Intelligence (UAI-97), San Francisco, CA, Morgan Kaufmann, 1997, pp. 282-293.

24. D u d a, R. O., P. E. Hart, D. G. S to r k. Pattern Classification, 2/e. New Delhi, Wiley-India Edition, 2007.

25. Porcu, Emilio, Montero, Jose-Marta, Schlather, Martin. Advances and Challenges in Space-Time Modelling of Natural Events. - In: Lecture Notes in Statistics. Vol. 207. Berlin, Heidelberg, Springer-Verlag, 2012.

26. Purdue University Cluster Software. https://engineering.purdue.edu/ bouman/ software/cluster/

27. Amitava, G., H. S. W. K. Pinnaduwa. A FORTRAN Program for Generation of Multivariate Normally Distributed Random Variables. - Computers \& Geosciences, Vol. 13, 1987, No 3, pp. 221-233. 